\title{
YouTube Videos as a Source of Information on Colorectal Cancer: Problem of the Correctness of the Contents
}

\author{
Beuy Joob $^{1}$ (D) Viroj Wiwanitkit ${ }^{2}$
}

Published online: 2 July 2020

(C) American Association for Cancer Education 2020

The author hereby would like to give a reflection regarding the recently published article entitled "YouTube Videos as a Source of Information on Colorectal Cancer: What Do Our Patients Learn?" in Journal of Cancer Education [1]. Sahin et al. reported that "YouTube might not be an education resource for CRC suited to every patient" [1]. A lack of good authoritative and comprehensive properties is mentioned [1]. Indeed, the YouTube videos become the easily assessed tool for self-education by the patients. The effect of freely available education type of videos on the patients is an interesting consideration in patient education. Most patients have the trend to attach to the contents displayed on the video and develop their practice according to the perceived content from the video [2]. The interesting consideration is on the reliability and correctness of the content in the video on cancer for the patients. Here, the authors tried to follow the methodology of Sahin et al. and identified the available YouTube videos giving colorectal information in Thai language which are the information resource for local Thai colorectal cancer patients. Using Google search to find the available video that has just been newly published during the first fortnight of October 2018, there are at least 78 YouTube videos as a source of information on colorectal cancer in Thai languages. No YouTube videos are published from a standard government medical center or medical college. All videos were published by private accounts; some are clearly identified as private companies (dietary supplement company 15 videos, private hospital 10 videos, traditional and alternative medicine clinic 10 videos, the remaining ones have unknown nature). Regarding the 35 videos with clear source that are from the

Beuy Joob

beuyjoob@hotmail.com

Private Academic Practice, Bangkok, Thailand

2 Dr. DY Patil University, Pune, India private business sector, all give the incorrect data including non-scientific properties of non-scientific approval diagnostic tool, alternative medicine regimen, dietary supplementation, and therapy. Also, a sale promotion session can be seen in all videos. It seems that the purpose of the available video usually has hidden agenda on business and the incorrect information on colorectal cancer might be purposively prepared aiming at sale purposes without ethical concern. The control of information available on YouTube videos seems to be difficult and there should be the role of the local government public health to have a good surveillance system to prevent fake content aimed at luring cancer patients to the unethical business. While the YouTube video is a good and fast way for patient education, the control of the correctness of the content that is freely assessed by the public is necessary. In conclusion, control over the content use of publicly available advertisingsupported resources for patient education is needed. There is also a requirement for legitimate medical centers, colleges, and organizations to produce evidence-based content. An important challenge is the limitation of online content which must be according to the free speech rights in the USA, where YouTube is based. The local government should take action for development of public resources. Collaborations with international cancer education organizations are encouraged.

\section{References}

1. Sahin AN, Sahin AS, Schwenter F, Sebajang H (2018) YouTube videos as a source of information on colorectal cancer: what do our patients learn? J Cancer Educ 34:1160-1166. https://doi.org/10. 1007/s13187-018-1422-9. [Epub ahead of print]

2. MacLeod MG, Hoppe DJ, Simunovic N, Bhandari M, Philippon MJ, Ayeni OR (2015) YouTube as an information source for femoroacetabular impingement: a systematic review of video content. Arthroscopy. 31(1):136-142

Publisher's Note Springer Nature remains neutral with regard to jurisdictional claims in published maps and institutional affiliations. 